\title{
Non-invasive cardiac output evaluation in postoperative cardiac surgery patients, using a new prolonged expiration-based technique
}

\author{
Alessia Mattei $\cdot$ Emiliano Schena $\cdot$ Stefano Cecchini · \\ Paola Proscia $\cdot$ Paola Saccomandi $\cdot$ Sergio Silvestri • \\ Massimiliano Carassiti
}

Received: 30 July 2013/Accepted: 4 February 2014/Published online: 22 February 2014

\begin{abstract}
The gold standard methods to measure cardiac output (CO) are invasive and expose the patient to high risks of various complications. The aim of this study is to assess an innovative non-invasive method for $\mathrm{CO}$ monitoring in mechanically ventilated patients after cardiac surgery and its agreement with values obtained by thermodilution technique. Continuous monitoring of respiratory gas concentrations and airflow allows the estimation of $\mathrm{CO}$ through a newly developed algorithm derived from a modified version of the Fick equation. It consists of two phases: the first involves measurements during steady breathing state, and the second starts when a sudden perturbation into the carbon dioxide elimination process is introduced by a prolonged expiration. This prospective clinical study involved thirty-five adult patients, undergone cardiac surgery. The measurements were performed in curarized and haemodynamically stable patients, during the post-surgery recovery in intensive care unit. The study protocol, which lasted $1 \mathrm{~h}$ for each patient, consisted of 20 measurements obtained by prolonged expiration-based method and 10 by thermodilution. The estimation of $\mathrm{CO}$ using the proposed method $\left(\mathrm{CO}_{\mathrm{K}}\right)$ agreed with the thermodilution $\left(\mathrm{CO}_{\mathrm{T}}\right)$ as demonstrated by: a low mean bias between $\mathrm{CO}_{\mathrm{K}}$ and $\mathrm{CO}_{\mathrm{T}}$ considering all patients (i.e., $-0.11 \mathrm{~L} \mathrm{~min}^{-1}$ ); a best fitting line having slope $=0.98$,
\end{abstract}

\footnotetext{
A. Mattei · P. Proscia - M. Carassiti ( ( )

Department of Anaesthesia, University School of Medicine Campus Bio-Medico, Via Alvaro del Portillo, 200, 00128 Rome, Italy

e-mail: m.carassiti@unicampus.it

E. Schena $\cdot$ S. Cecchini $\cdot$ P. Saccomandi $\cdot$ S. Silvestri Research Laboratory of Measurements and Biomedical Instrumentation, University Campus Bio-Medico, Via Alvaro del Portillo, 200, 00128 Rome, Italy
}

$\mathrm{r}=0.81, p<0.0001$; the lower and upper limits of agreement were -0.77 and $+0.54 \mathrm{~L} \mathrm{~min}^{-1}$, respectively. $\mathrm{CO}_{\mathrm{K}}$ shows a mean percentage error of $34 \%$. In stable mechanically ventilated patients, undergone cardiac surgery, the proposed method is reliable if compared to the thermodilution. Considering the non-invasivity of the technique, further evaluations of its performances are encouraged.

Keywords Cardiac output - Non-invasive method · Fick method · Thermodilution · Prolonged expiration

\section{Introduction}

The gold standards for CO measurement, the Fick method and the pulmonary artery thermodilution [1], are both invasive and expose the patient to risk of various complications [2].

In the last decade several minimally invasive or noninvasive techniques for $\mathrm{CO}$ monitoring have been developed and tested with the aim to reduce the risks for the patient. The widespread use of these methods in clinical practice has been delayed for the high costs of the devices and concerns about accuracy, precision, and reproducibility [3].

Some techniques based on the Fick method, employ the pulmonary blood flow (PBF) to estimate $\mathrm{CO}$ [4-7]. We proposed a non-invasive method, already described in our previous study [8], which calculates $\mathrm{CO}$ applying the differential application of the Fick principle to the $\mathrm{CO}_{2}$. The expired gas content was monitored during both normal breathing and prolonged expiration in order to noninvasively estimate $\mathrm{PaCO}_{2}$ and $\mathrm{PvCO}_{2}$. This estimation was based on the algorithm proposed by Kim et al. [9] and 
provides two of the respiratory parameters, which can be estimated from a change in the breathing pattern [10].

Therefore, this study aims to reinforce our previous findings, by performing the Kim-based technique on 35 mechanically ventilated patients, and to assess the agreement between the results estimated by this non-invasive technique and the values obtained by thermodilution. This concordance study was carried out in post cardiac surgery patients.

\section{Materials and methods}

The study was approved by the institutional Ethics Committee of the University Hospital Campus Bio-Medico in Rome (Prot. N. 19/2011 ComEt CBM) and written informed consent was obtained from each patient. The trial (NCT01714258) was registered at ClinicalTrials.gov.

Thirty-five adult patients who underwent cardiac surgery for coronary artery bypass or valvular surgery were enrolled in this study from March 2011 to February 2012.

Enrolment criteria were: (1) insertion of a Swan-Ganz catheter; (2) stable heamodynamics in the intensive care unit.

We excluded candidates who: were haemodynamically unstable and required high doses of vasoactive medications ( $>5 \mu \mathrm{g} \mathrm{kg}^{-1} \mathrm{~min}^{-1}$ of dopamine or dobutamine), crystalloids or colloids to maintain blood pressure, or inspired oxygen concentration higher than $60 \%$ in the postopera-tive period; patients affected by central nervous system disorders, or whom might have been adversely affected by induced hypercapnia (risk of severe pulmonary hypertension or increased intracranial pressure). We also excluded patients with severe tricuspid regurgitation, since it interferes with the accuracy of thermodilution $\mathrm{CO}$ measurement or with an ejection fraction lower than $40 \%$ and patients with significant lung disease in whom end tidal $\mathrm{CO}_{2}$ may not represent $\mathrm{PaCO}_{2}$.

Haemodynamic measurements were performed in the ICU, after waiting $1-3 \mathrm{~h}$ for haemodynamic to stabilize after surgery.

All patients recruited in this prospective study had a 7.5F flow-directed thermistor-tipped pulmonary artery catheter (Edwards Lifesciences), were intubated and ventilated by Servo-I ventilators (Maquet Gmbh\& Co. KG). Arterial pressure monitoring was standard in all patients and obtained by either radial or femoral catheterization. At the time of admission to the intensive care unit, the ventilator was set to synchronized intermittent mandatory ventilation mode, with an inspired tidal volume of $8 \mathrm{~mL} \mathrm{~kg}^{-1}$; respiratory rate of $10-12 \mathrm{bpm}$. The $\mathrm{F}_{\mathrm{I}} \mathrm{O}_{2}$ (inspiratory fraction of oxygen) was adjusted to maintain a $\mathrm{PaO}_{2}$ (arterial partial pressure of oxygen) greater than $100 \mathrm{mmHg}$. Baseline
PEEP was set at $5 \mathrm{~cm} \mathrm{H}_{2} \mathrm{O}$ and remained unchanged during the study protocol.

The patients, in supine position, were kept sedated with continuous intravenous injection of propofol (1-3 $\mathrm{mg} \mathrm{kg}^{-1} \mathrm{~h}^{-1}$ ), and curarized with bolus administration of vecuronium bromide $(10 \mathrm{mg})$. Once all these conditions were established, the measurement protocol could start.

\subsection{Cardiac output measurement by thermodilution $\left(\mathrm{CO}_{\mathrm{T}}\right)$}

Standard pulmonary artery thermodilution CO measurements (Vigilance, Edwards Lifesciences) were carried out using $10 \mathrm{~mL}$ bolus injections of $5 \%$ glucose solution at room temperature $\left(21-24^{\circ} \mathrm{C}\right)$. The averaged values for three or four injections were used to determine each cardiac output.

Each time a $\mathrm{CO}_{\mathrm{T}}$ measurement was taken, the most recent cardiac output measured by the prolonged expiration technique was documented.

Injection time was always shorter than $4 \mathrm{~s}$ to reduce any effect of varying injection rates on calculations and we standardized the timing of bolus injection after the first half of the expiratory phase. The same operator executed all the injections. The morphology of thermodilution curves was always monitored to detect artefacts. All the curves of the changes in temperature in the pulmonary artery were inspected for irregularities and accepted/rejected before the results were displayed on the monitor. Aborted attempts were replaced by a new injection.

\subsection{Cardiac output measured by prolonged expiration technique $\left(\mathrm{CO}_{\mathrm{K}}\right)$}

The non-invasive technique for measuring $\mathrm{CO}$ using a modified Fick principle [11] applied to $\mathrm{CO}_{2}$, already described in detail [8], allows an estimation of the $\mathrm{CO}$ by calculating the PBF from the mass conservation of $\mathrm{CO}_{2}$.

By considering a linear $\mathrm{CO}_{2}$ dissociation curve, Eq. 1 can be expressed as follows:

$$
P B F=\frac{\dot{V}_{\mathrm{CO}_{2}}}{S \cdot\left(P_{V} \mathrm{CO}_{2}-P_{a} \mathrm{CO}_{2}\right)}
$$

where $\mathrm{PaCO}_{2}$ is the partial pressure of $\mathrm{CO}_{2}$ in the arterial blood, $\mathrm{PvCO}_{2}$ is the partial pressure of $\mathrm{CO}_{2}$ in the venous blood, $\mathrm{S}$ is the $\mathrm{CO}_{2}$ solubility in blood where $\dot{V}_{\mathrm{CO}_{2}}$ is the amount of $\mathrm{CO}_{2}$ produced by the subject in unit of time.

Considering that $\mathrm{CO}$ represents the whole amount of blood pumped by the right ventricle, whilst PBF is the fraction of $\mathrm{CO}$ that participate actively to the pulmonary exchange into open alveoli, $\mathrm{CO}$ can be calculated using PBF as follows: 


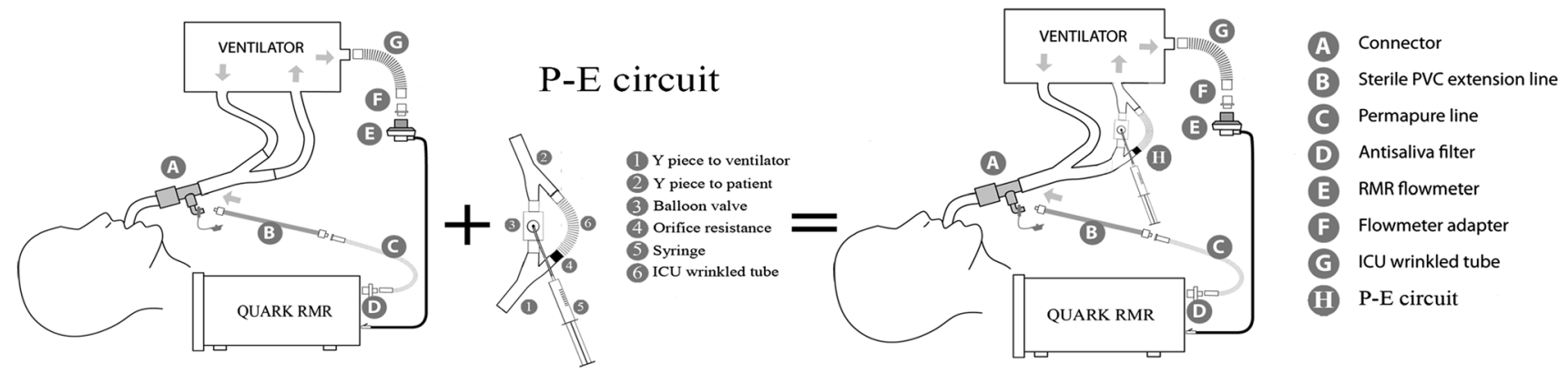

Fig. 1 Schematic representation of the components used to monitor $\mathrm{CO}$ on mechanically ventilated patients. Left side circuit used during normal breathing; right side circuit used during prolonged expiration; a detail of the $\mathrm{P}-\mathrm{E}$ circuit is also reported

$C O=\frac{P B F}{1-F}$

where $F$ is the shunting fraction, the ratio between the amount of blood, which does not participate to the alveolar gas exchange, and the amount of blood pumped by the right ventricle during heart contraction. $\mathrm{F}$ is calculated using $\mathrm{FIO}_{2}$ and $\mathrm{PaO}_{2}$, obtained from arterial blood-gas analysis. Iso-shunt diagrams are used to obtain $\mathrm{F}$ : these diagrams are a series of continuous curves showing the relationship between $\mathrm{PaO}_{2}$ and $\mathrm{FIO}_{2}$ for different shunting fractions [12].

As already specified in our previous study, in order to estimate $\mathrm{CO}$, we proposed a method based on the analysis of the respiratory gas content during both normal breathing and prolonged expiration. This method was used to calculate the denominator of Eq. 1 without any invasive measurement [8]. The system, schematically reported in Fig. 1, was introduced at this purpose: on the left side, a normal breathing circuit, with a gas sampling line, is represented; on the right side, an additional circuit (P-E circuit) is added to the same breathing circuit with the function to induce the prolonged expiration. The P-E cir-cuit is a custom made 2-branch element: in one branch, an orifice pneumatic resistance is inserted to increase the expiratory time, in the second one a balloon valve. When the valve is open, the majority of the expired flow goes through it; when it is closed, the whole expiratory flow goes through the orifice resistance inducing the prolonged expiration.

During the prolonged expiration, the expiratory pause hold' key of the ventilator was always pushed to avoid the delivery of an inspiratory act and the risk of volutrauma and barotrauma for the patient. At the end of the manoeuvre, the valve's balloon was deflated and the ven-tilator unlocked: then the new inspiratory act can be delivered.

As reported in Fig. 1, breathing gases were sampled from the breathing circuit using a sampling line (B) and analysed by a metabolic monitor (Quark RMR, Cosmed srl), which measures the partial pressure of $\mathrm{CO}_{2}$ and $\mathrm{O}_{2}$ in the expiratory gas. These data were used to estimate $\mathrm{PaCO}_{2}$ and $\mathrm{PvCO}_{2}$, by an algorithm running in $\mathrm{LabVIEW}^{\circledR}$ environment (National Instruments Corporation), as described in detail in [8].

The manoeuvre to induce the prolonged expiration was executed 20 times for each patient: it lasted about $15 \mathrm{~s}$, with a 2 min pause between consecutive manoeuvres, to allow the recovering of steady conditions by the subject, and the assessment of the subject's gas exchange.

Before inducing each manoeuvre of prolonged expiration, we assessed the stability of haemodynamic conditions of the patients. No relevant change in haemodynamic status during the study protocol was documented.

In summary, the timing of the trial can be described as follows. The determination of $\mathrm{CO}_{\mathrm{T}}$ was performed ten times: 4 times at steady condition, 3 times after a sequence of $10 \mathrm{CO}_{\mathrm{K}}$ and 3 times at the end of the session, after other $10 \mathrm{CO}_{\mathrm{K}}$ measurements; the total duration of the protocol was about $60 \mathrm{~min}$. Each $\mathrm{CO}_{\mathrm{K}}$ was compared with the most recent $\mathrm{CO}_{\mathrm{T}}$ value.

\subsection{Statistical analysis}

Descriptive statistics, which included mean and standard deviation (SD), were calculated for stature, weight and age and a comparison between male and female mean values was performed using the $t$ test. Significant level was set at $p<0.01$.

The correlation between $\mathrm{CO}$ estimated using the Kim non-invasive approach $\left(\mathrm{CO}_{\mathrm{K}}\right)$ and the thermodilution $\left(\mathrm{CO}_{\mathrm{T}}\right)$ was evaluated considering all trials performed on each patient, and was examined by simple regression analysis using Pearson's correlation coefficient (r).

Furthermore, the agreement between $\mathrm{CO}_{\mathrm{K}}$ values and the values of $\mathrm{CO}_{\mathrm{T}}$ was performed using two approaches: (1) Bland-Altman method, recommended for comparing the agreement between two approaches of clinical measurements; (2) differences between $\mathrm{CO}_{\mathrm{K}}$ and $\mathrm{CO}_{\mathrm{T}}$ were 
Table 1 Descriptive statistical analysis

\begin{tabular}{|c|c|c|c|c|c|c|c|}
\hline \multirow[t]{2}{*}{ Variable } & \multicolumn{3}{|l|}{ Males } & \multicolumn{3}{|c|}{ Females } & \multirow[t]{2}{*}{$p$ value } \\
\hline & Total & Mean & SD & Total & Mean & SD & \\
\hline Age (years) & 21 & 66.8 & 11.7 & 14 & 70.7 & 10 & $p>0.01$ \\
\hline Weight (kg) & 21 & 81.2 & 8.8 & 14 & 66.3 & 13 & $p<0.01$ \\
\hline Stature $(\mathrm{cm})$ & 21 & 174 & 5.5 & 14 & 161 & 7.6 & $p<0.01$ \\
\hline \multicolumn{8}{|c|}{ Haemodynamic results } \\
\hline $\mathrm{CO}_{\mathrm{T}}\left(\mathrm{L} \min ^{-1}\right)$ & 21 & 4.13 & 1.1 & 14 & 3.15 & 0.68 & $p<0.01$ \\
\hline $\mathrm{CO}_{\mathrm{K}}\left(\mathrm{L} \min ^{-1}\right)$ & 21 & 4.01 & 1.1 & 14 & 3.07 & 0.75 & $p<0.01$ \\
\hline $\operatorname{PBF}\left(\mathrm{L} \min ^{-1}\right)$ & 21 & 3.58 & 0.98 & 14 & 2.80 & 0.67 & $p<0.01$ \\
\hline $\mathrm{F}(\%)$ & 21 & 10.8 & 7.6 & 14 & 8.7 & 6.3 & $p>0.01$ \\
\hline
\end{tabular}

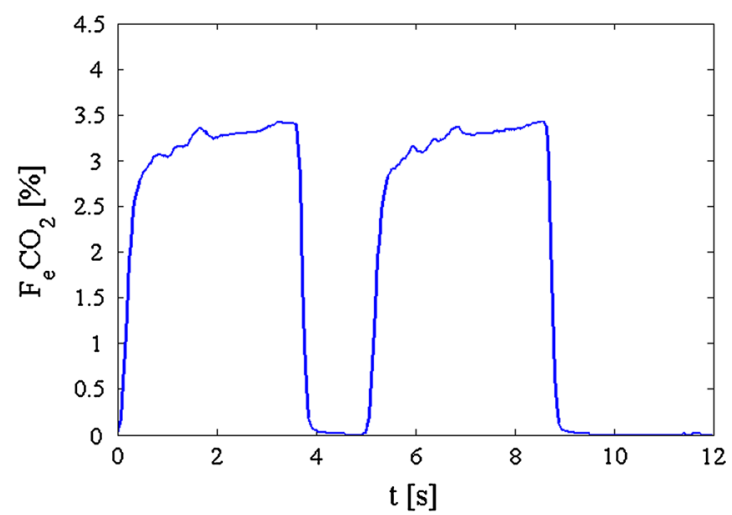

Fig. 2 Expiratory fraction of $\mathrm{CO}_{2}$ : left side trend of $\mathrm{CO}_{2}$ during two normal expirations; right side trend of $\mathrm{CO}_{2}$ during a single prolonged expiration

calculated for all measurements performed on each patient and presented by histograms and cumulative distribution plots. Also the percentage difference between the mean values of $\mathrm{CO}_{\mathrm{K}}$ and $\mathrm{CO}_{\mathrm{T}}$ are calculated for all patients.

All the statistics were analysed in the MatLAB ${ }^{\circledR}$ (MathWorks Inc.) environment.

\section{Results}

By performing the Kim-based technique on 35 mechanically ventilated patients, undergone cardiac surgery and whose characteristics (mean age 68.0 with SD of 11.3 years) are listed in Table 1 , we assessed the agreement between the results estimated by this non-invasive technique and the values obtained by thermodilution.

The protocol included a prolonged expiration manoeuvre, which was well tolerated by all patients.

Typical trends of $\mathrm{CO}_{2}$ expiratory fraction $\left(\mathrm{F}_{\mathrm{e}} \mathrm{CO}_{2}\right)$ with and without prolonged expiration are reported in Fig. 2.

Figure 3 shows the correlations between $\mathrm{CO}_{\mathrm{T}}$ and $\mathrm{CO}_{\mathrm{K}}$, considering all patients: the best fitting line was $\mathrm{CO}_{\mathrm{K}}=$ $0.9783 \cdot \mathrm{CO}_{\mathrm{T}}-0.0520$, the correlation coefficient was $\mathrm{r}=$ 0.81 , and a significant correlation was found between $\mathrm{CO}_{\mathrm{T}}$ and $\mathrm{CO}_{\mathrm{K}}(\rho<0.0001)$.

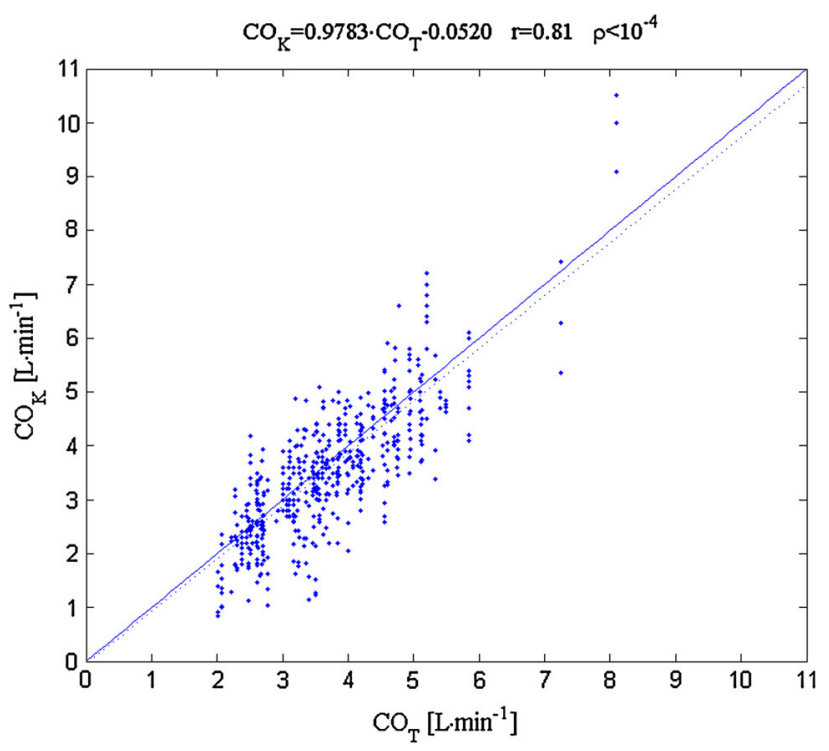

Fig. 3 Values of $\mathrm{CO}_{\mathrm{K}}$ as a function of $\mathrm{CO}_{\mathrm{T}}$. The best fitting line (dashed line) and the line of equality (continuous line) are also shown

The agreement between $\mathrm{CO}_{\mathrm{K}}$ and $\mathrm{CO}_{\mathrm{T}}$ is shown in different ways in Fig. 4.

Considering all the trials for each subjects, $\triangle \mathrm{CO}$ was always less than $2.5 \mathrm{~L} \mathrm{~min}^{-1}$. 

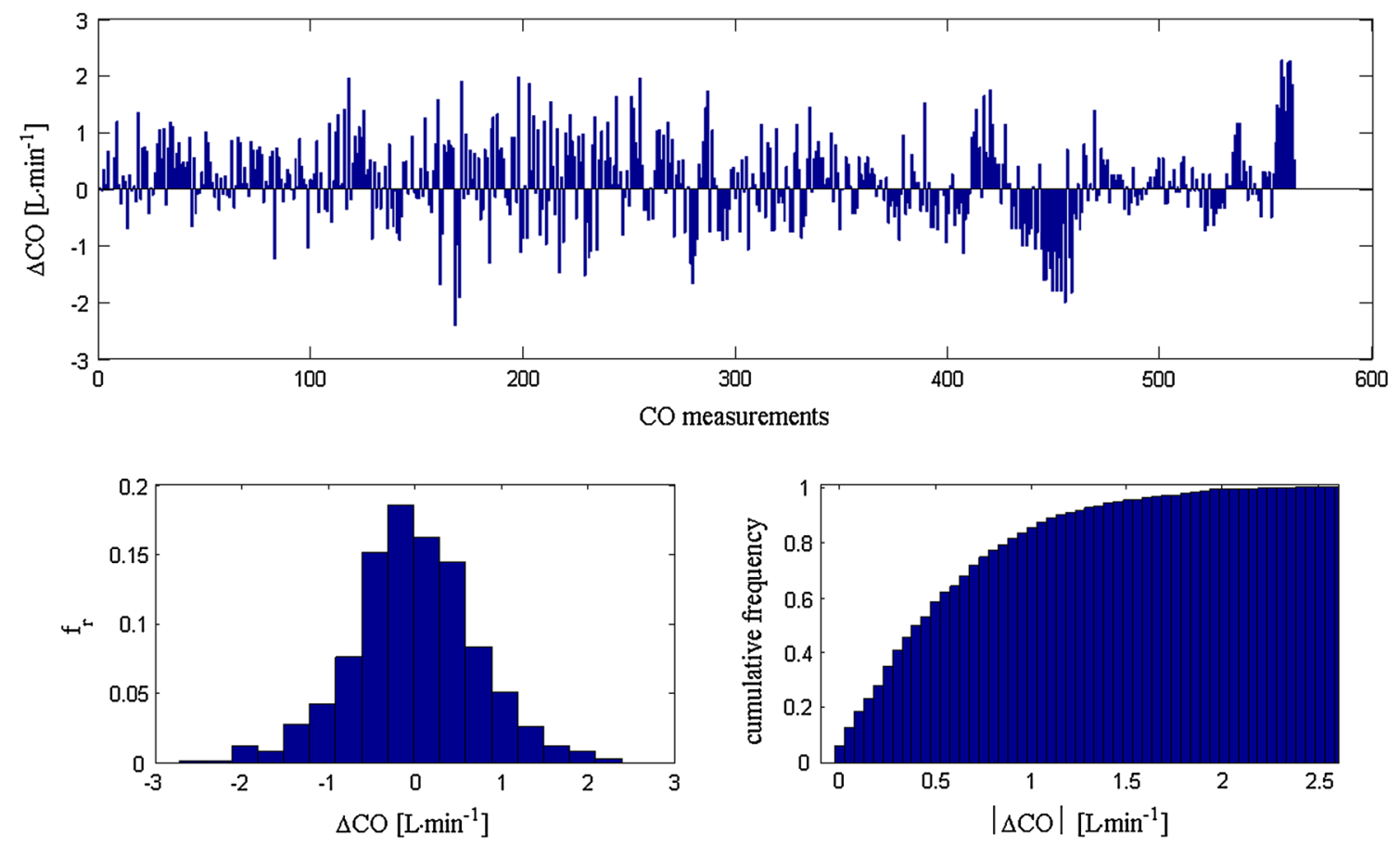

Fig. 4 Values of $\Delta \mathrm{CO}=\mathrm{CO}_{\mathrm{T}}-\mathrm{CO}_{\mathrm{K}}$, their histogram and cumulative distribution plot in all trials

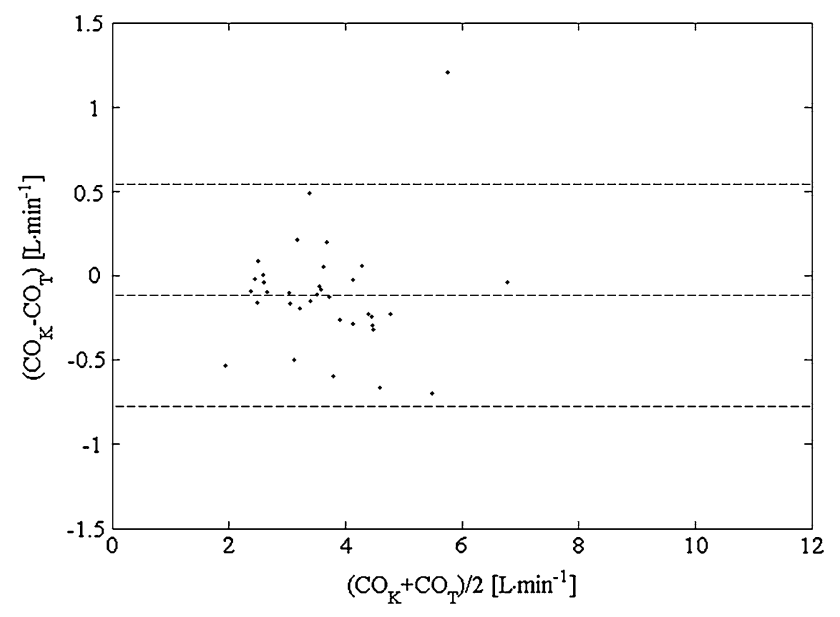

Fig. 5 Bland-Altman plot showing the comparison between $\mathrm{CO}_{\mathrm{T}}$ and $\mathrm{CO}_{\mathrm{K}}$, the three dotted lines represent the mean bias between the two methods and the mean $\pm 1.96 \cdot \mathrm{SD}$ of $\Delta \mathrm{CO}$

These differences were greater than $1 \mathrm{~L} \mathrm{~min}^{-1}$ in less than $13 \%$ of all trials, the 95th percentile is reached at $1.45 \mathrm{~L} \mathrm{~min}^{-1}$, and in about $50 \%$ of the trials the difference ranged in $\pm 0.4 \mathrm{~L} \mathrm{~min}^{-1}$.

The Bland-Altman plot, reported in Fig. 5, represents the rate of agreement between $\mathrm{CO}_{\mathrm{T}}$ and $\mathrm{CO}_{\mathrm{K}}$. The mean bias for each patient was about $-0.11 \mathrm{~L} \mathrm{~min}^{-1}$.

The mean values of $\mathrm{CO}$ obtained using the two approaches and their percentage difference, expressed as $\Delta \mathrm{CO} \%=100 \cdot\left(\mathrm{CO}_{\mathrm{K}}-\mathrm{CO}_{\mathrm{T}}\right) / \mathrm{CO}_{\mathrm{T}}$ are reported in Fig. 6.
The mean values of $\mathrm{CO}_{\mathrm{T}}$ and $\mathrm{CO}_{\mathrm{K}}$ presented a good agreement: their percentage difference was lower than $7 \%$ in 28 patients $(80 \%)$. In this sample of patients, mean $\mathrm{CO}$ estimated by invasive approach was $4.13 \mathrm{~L} \mathrm{~min}^{-1}$ for males and $3.15 \mathrm{~L} \mathrm{~min}^{-1}$ for females; mean $\mathrm{CO}$ estimated by our non-invasive approach was $4.01 \mathrm{~L} \mathrm{~min}^{-1}$ for males and 3.07 $\mathrm{L} \min ^{-1}$ for female. In both cases $\mathrm{CO}_{\mathrm{K}}$ slightly underestimated $\mathrm{CO}_{\mathrm{T}}$ values, as also shown in the BlandAltman plot (Fig. 5). Furthermore, a comparison between male and female mean values of $\mathrm{CO}$ was performed using the $t$ test: both thermodilution and Kim methods showed CO values lower in females than in males, and, the dif-ference was significant $(p \leq 0.01)$.

Furthermore, the percentage error, expressed as $E \%=100 \cdot(1.96 \cdot S D / \overline{C O})$, was calculated, where $\mathrm{SD}$ is the standard deviation of the $\mathrm{CO}$ values obtained for each patient, and $\overline{C O}$ is the mean value of $\mathrm{CO}$ obtained for each patient. Considering all the patients, E\% shows a mean value of 34 and $25 \%$ for the non-invasive method and for thermodilution, respectively.

In order to evaluate the effectiveness of the proposed method on a shorter timeframe and to give a closer view on the applicability of the system, it is possible to consider a procedure time of $10 \mathrm{~min}$. Over $10 \mathrm{~min}, 3$ trials of $\mathrm{CO}_{\mathrm{T}}$ and 5 trials of $\mathrm{CO}_{\mathrm{K}}$ are executed. The comparison between the mean of the first three values of $\mathrm{CO}_{\mathrm{T}}$ and the first five values of $\mathrm{CO}_{\mathrm{K}}$ shows an increase of the mean bias respect on the standard trial $\left(-0.36\right.$ vs $\left.-0.11 \mathrm{~L} \mathrm{~min}^{-1}\right)$. Considering all the patients, the SD of the bias between the two 

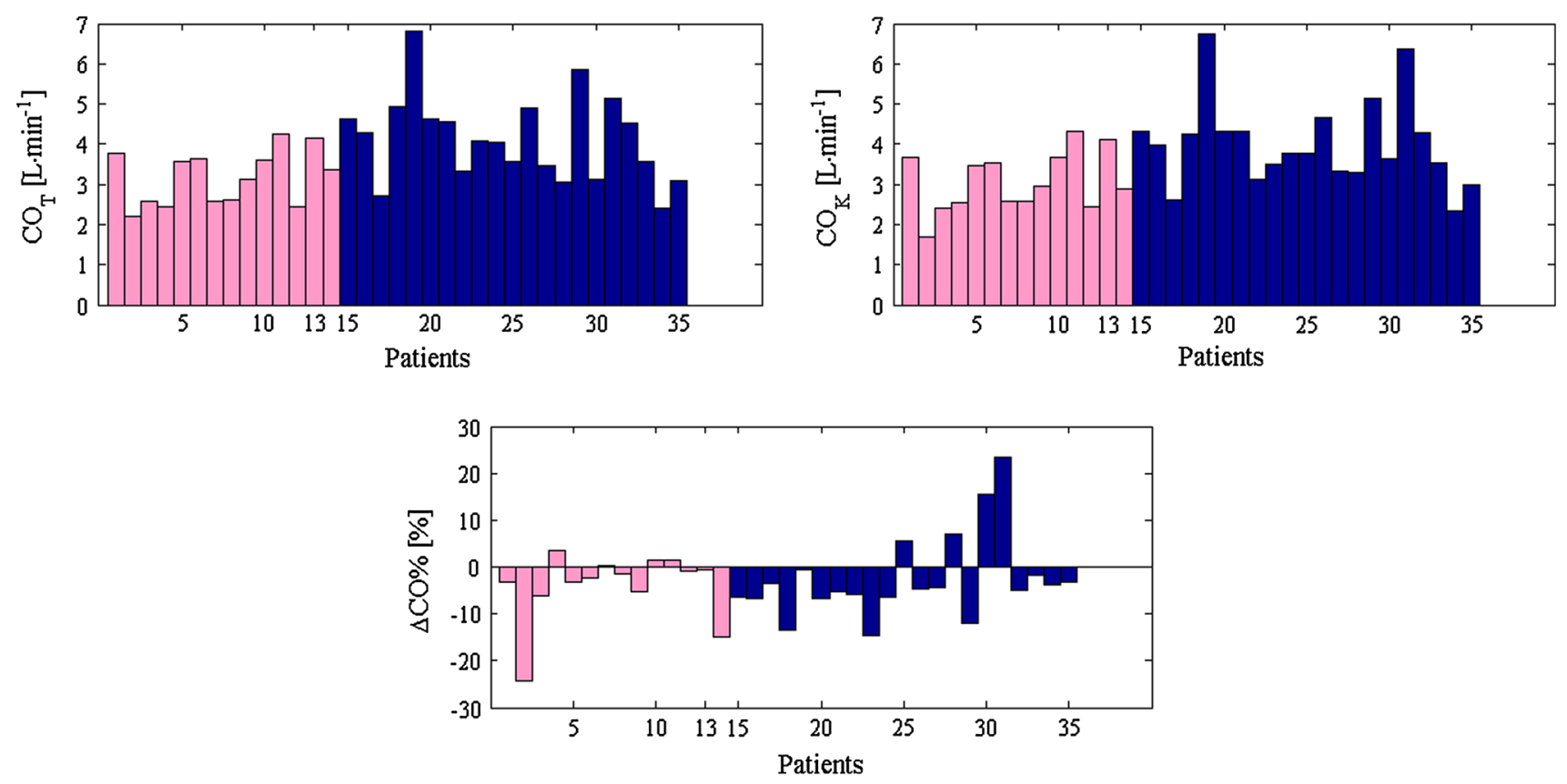

Fig. 6 Mean values of $\mathrm{CO}_{\mathrm{T}}$ and $\mathrm{CO}_{\mathrm{K}}$ for each patient and their percentage difference (pink bars females, blue bars males)

methods results $0.40 \mathrm{~L} \mathrm{~min}^{-1}$, and the mean value of the percentage errors results $27 \%$.

\section{Discussion}

The main findings of the present work can be resumed as follows: $\mathrm{CO}_{\mathrm{K}}$ and $\mathrm{CO}_{\mathrm{T}}$ showed good correlation $(\mathrm{r}>0.80)$; the slope of the best fitting line was slightly lower than the line of equality (0.98); the mean bias con-sidering all patients showed a negligible underestimation $(-2.9 \%)$. These values were in line with the results reported by Peyton and Chong [3]. In the process of evaluating the accuracy and precision of a novel technique to measure cardiac output, this must be compared with a reference standard and the percentage error of agreement is the most frequent criterion of acceptability. In this study $\mathrm{CO}_{\mathrm{K}}$ values showed a percentage difference with $\mathrm{CO}_{\mathrm{T}}$ of $30 \%$ lower than the limit recommended [13], in all patients: in particular, the maximum value was about $25 \%$ and in 28 patients was lower than $7 \%$ (Fig. 5).

Several previous studies investigated a prolonged expiration method $[9,14]$, allowing a non-invasive estimation of $\mathrm{PvCO}_{2}$ and $\mathrm{PaCO}_{2}$, according to the Fick method. None of these studies has ever involved mechanically ventilated patients or used thermodilution as a reference method. In this work we proceeded from the results presented in a previous study [8], in which, for the first time we performed an in vivo validation of the method on mechanically ventilated patients, and compared it with the thermodilution.
This study also confirmed that the non-invasive Fick method underestimates thermodilution data. The underestimation obtained with our technique was lower than the consistent underestimation reported by Killick and Parkin [7]: they showed a bias of $-0.60 \pm 0.87 \mathrm{~L} \mathrm{~min}^{-1}$ versus our result of $-0.11 \pm 0.66 \mathrm{~L} \mathrm{~min}^{-1}$.

The CO underestimation can be partially explained by the limitations intrinsic to the non-invasive method. A crucial aspect in the differential modification of the Fick principle is that the venous content of carbon dioxide $\left(\mathrm{CvCO}_{2}\right)$ remains constant. A potential limitation of the Kim algorithm is the difficulty in the assessment of $\mathrm{PvCO}_{2}$ changes during prolonged expiration. During prolonged expiration and similarly to the rebreathing period, there could be a rise in $\mathrm{CvCO}_{2}$, which can be caused by a $\mathrm{CO}_{2}$ build-up in the blood, related to re-circulation. Even this small rise may contribute to increase arterial $\mathrm{CO}_{2}$ content $\left(\mathrm{CaCO}_{2}\right)$ and lead to underestimate $\mathrm{CO}$. Another potential source of error can be associated to the underestimation of $\mathrm{CO}_{2}$ values measured at the mouth, probably caused by the contamination of gases during expiration through dead space, and the storage of expired $\mathrm{CO}_{2}$ in the lungs. Due to the effect of dead-space, $\mathrm{PetCO}_{2}$ is lower than $\mathrm{PaCO}_{2}$ leading to an overestimation in the change in $\mathrm{CaCO}_{2}$, and to a consequent underestimation of the cardiac output measurement, proportional to the increase in dead-space.

In addition, the calculation of the final value of cardiac output results from an estimation of shunt fraction. The estimation of PBF only (without considering F) increases the percentage difference between the proposed method and the 
thermodilution: it becomes $-13.4 \%$ for males (PBF $=3.58 \mathrm{~L} \mathrm{~min}^{-1}$ and $\mathrm{CO}_{\mathrm{T}}=4.13 \mathrm{~L} \mathrm{~min}^{-1}$ ) and $-11.0 \%$ for females $\left(\mathrm{PBF}=2.80 \mathrm{~L} \mathrm{~min}^{-1}\right.$ and $\mathrm{CO}_{\mathrm{T}}=3.15 \mathrm{~L}$ $\left.\min ^{-1}\right)$. Therefore the use of $\mathrm{F}$ allows improving the agreement between $\mathrm{CO}_{\mathrm{K}}$ and $\mathrm{CO}_{\mathrm{T}}(-2.9 \%$ for males and $-2.6 \%$ for females).

As previous studies underlined, the non invasive techniques, which estimate the shunt fraction by the iso-shunt diagram, tend to give a lower shunt fraction values than those resulting from the shunt equation [15]. In patients with large intrapulmonary shunt, there could be a greater underestimation of $\mathrm{CO}$ using the non invasive technique. In general these patients exhibited a hyperdynamic state and the loss of hypoxic pulmonary vasodilatation have led to an increased shunt.

However, it must be considered that the slight underestimation obtained with our technique may be explained by the overestimate of $\mathrm{CO}$ by thermodilution. Over the years, concerns about the accuracy of thermodilution have led studies, which compared it with more accurate tech-niques. These studies showed a tendency to over-estimate $\mathrm{CO}$ when compared with ultrasound transit-time flowmetry of the ascending aorta using an ultrasonic flow probe [16] and dye dilution with indocyanine green [17]. Moreover, thermodilution can be affected by injection technique and changes in injectate temperature.

The great advantage of this technique we propose stands in its repeatability without operating invasive procedures on the patient. Nevertheless, the following hypotheses, on which the proposed method is based, have to be respected [8]: all $\mathrm{CO}_{2}$ participating in the alveolar exchange is released, the concentrations and fractions of the expired gas in the expiratory plateaus are equal to those of the alveolar gas, considering the hypothesis of perfect mixing, and $\mathrm{CO}$ is not altered by the prolonged expiration.

This technique is characterized by five main limitations: (1) the prolonged expiration method may not be suitable for subjects with obstructive pulmonary disease, since a representative mixed alveolar sample cannot be obtained with any degree of certainty in these subjects; (2) a large increase in pulmonary dead-space ventilation and intrapulmonary shunt is commonly caused by intraoperative manipulation of the lung and consequent collapse of the parenchyma and the use of medications which inhibits pulmonary hypoxic vasoconstriction; (3) this method does not allow beat to-beat estimates of $\mathrm{CO}$, similarly to thermodilution; (4) the method requires stable tidal volumes to permit $\mathrm{CO}_{2}$ elimination measurement and therefore it cannot be applied in patients sedated in pressure support modes; and (5) long measuring time. An average over multiple estimations of COK is needed to obtain acceptable agreement between the two techniques. Considering that a timeframe of $1 \mathrm{~h}$ allows reducing the mean bias $(-0.11 \mathrm{vs}$
$-0.36 \mathrm{~L} \mathrm{~min}-1$ over a timeframe of $10 \mathrm{~min}$ ) without relevant changes in the percentage error, an important goal for future research would be the assessment of the optimal compromise between the accuracy and the measuring time.

In summary, this new technique for the measurement of $\mathrm{CO}$, which uses a prolonged expiration manoeuvre, offers promising results. The possible reasons of the slight underestimation of the cardiac output values obtained by thermodilution have been discussed. The good results showed by this study and the advantages related to the noninvasivity of the method prove its reliability, encourage to carry out further investigation and to introduce solutions aiming to improve the $\mathrm{CO}$ estimation on unstable patients.

Acknowledgments The authors gratefully acknowledge Cosmed s.r.l. (Rome, Italy) for the precious support provided.

Conflict of interest The authors declare that they have no conflict of interest.

\section{References}

1. Norman RA, Johnson RW, Messinger JE, Sohrab B. A continuous cardiac output computer based on thermodilution principles. Ann Biomed Eng. 1989;17:61-73.

2. Rivers E, Nguyen B, Havstad S. Early goal directed therapy in the treatment of sepsis and severe shock septic. N Engl J Med. 2001;345:1368-77.

3. Peyton PJ, Chong SW. Minimally invasive measurement of cardiac output during surgery and critical care: a meta-analysis of accuracy and precision. Anesthesiology. 2010;113:1220-35.

4. Gedeon A, Forslund L, Hedenstierna G, Romano E. A new method for noninvasive bedside determination of pulmonary blood flow. Med Biol Eng Comput. 2002;17:313-21.

5. Peyton PJ, Thompson D, Junior P. Non-invasive automated measurement of cardiac output during stable cardiac surgery using a fully integrated differential CO2 Fick method. J Clin Monit Comput. 2008;22:285-92.

6. Haryadi DG, Orr JA, Kuck K, McJames S, Westenskow DR. Partial $\mathrm{CO}_{2}$ rebreathing indirect Fick technique for non-invasive measurement of cardiac output. J Clin Monit Comput. 2000;16: 361-74.

7. Killick CJ, Parkin WG. Non-invasive cardiac output measurement using a fast mixing box to measure carbon dioxide elimination. Anaesth Intensive Care. 2008;36:665-73.

8. Cecchini S, Schena E, Notaro M, Carassiti M, Silvestri S. Noninvasive estimation of cardiac output in mechanically ventilated patients: a prolonged expiration method. Ann Biomed Eng. 2012;40:1777-89.

9. Kim TS, Rahn H, Fahri LE. Estimation of true venous and arterial $\mathrm{P}_{\mathrm{CO} 2}$ by gas analysis of a single breath. J Appl Physiol. 1985;5:39-48.

10. Mitchell RR. A deep breath method for noninvasive estimation of cardiopulmonary parameters. J Clin Monit Comput. 1988;5: 53-64.

11. Fick A. Uber die messung des blutquantums in den hertzventrikeln. Sitzungsberichte der Physiologisch Medizinosche Gesellschaft zu Wuerzburg. 1870;2:16.

12. Jaffe MB. Partial $\mathrm{CO} 2$ rebreathing cardiac output operating principles of the NICO TM system. J Clin Monit Comput. 1999;15:387-401. 
13. Critchley LAZ, Critchley LAZ. A meta-analysis of studies using bias and precision statistics to compare cardiac output measurement techniques. J Clin Monit Comput. 1999;15:85-91.

14. Godfrey S. Manipulation of the indirect Fick principle by a digital computer program for the calculation of exercise physiology results. Respiration. 1970;27:513-32.

15. Odenstadt H, Stenqvist O, Lundin S. Clinical evaluation of a partial $\mathrm{CO} 2$ rebreathing technique for cardiac output monitoring in critically ill patients. Acta Anaesthesiol Scand. 2002;46:152-9.
16. Botero M, Kirby D, Lobato EB, Staples ED, Gravenstein N. Measurement of cardiac output before and after cardio-pulmonary bypass: comparison among aortic transit-time ultrasound, thermodilution, and noninvasive partial $\mathrm{CO} 2$ rebreathing. J Cardiothorac Vasc Anesth. 2004;18:563-72.

17. Russell AE, Smith SA, West MJ, Aylward PE, McRitchie RJ, Hassam RM, et al. Automated non-invasive measurement of cardiac output by the carbon dioxide rebreathing method: comparisons with dye dilution and thermodilution. Br Heart J. 1990;63:195-9. 